Angela Störl

Die Konferenz wurde von Prof. Dr. med. Reinhard Saller, Direktor des Institutes für Naturheilkunde des UniversitätsSpitals Zürich, eröffnet (Abb. 1, 2). Der Komplementärmediziner gab einen Überblick über die Grundlagen der Phytotherapie, wie sie die Tibetische Medizin verwendet. Dr. Herbert Schwabl, Geschäftsführer der Padma AG und Präsident des SVKH (Schweizerischer Verband für komplementärmedizinische Heilmittel), und Prof. Dr. Florian Überall beleuchteten den Netzwerkcharakter tibetischer Vielstoffgemische sowie einen neuen netzwerkbasierten Forschungsansatz. Die Pflanzenrezepturen der Tibetischen Medizin bestehen aus drei bis zu 50 verschiedenen Komponenten. Forschungsergebnisse zeigen, dass sie vielfältige Impulse setzen und auf unterschiedliche Stoffwechselebenen gleichzeitig einwirken. Dadurch sind sie meist sehr gut verträglich und haben ein breites Wirkprofil. Dies ist vor allem in Anbetracht der Zunahme der Multimorbidität wichtig, die ein zentrales Problem für unsere Bevölkerung und eine grosse Belastung für das Gesundheitssystem darstellt, erklärte Prof. Saller. Lifestyle-Erkrankungen - nicht übertragbare Krankheiten - wie Diabetes, Krebs oder Herz-Kreislauf-Beschwerden sind auf dem Vormarsch und in den Industrienationen für $86 \%$ der Todesfälle verantwortlich. Gerade hier eröffnet die Systembiologie neue Blickwinkel, konstatierte Dr. Herbert Schwabl. «Der Organismus muss als komplexes Netzwerk mit verschiedenen Interaktionen und hierarchischen Ebenen verstanden werden.» Studien ergaben, dass tibetische Vielstoffgemische nicht bloss

\title{
Fortbildung
}

Schweiz Z Ganzheitsmed 2012;24:355-357

DOI: 10.1159/000345449

\section{Tibetische Arzneimittel in der modernen Medizin: Neue Wege bei Multimorbidität}

Durch ein optimiertes Krankheitsmanagement leben die Menschen heute länger. Akute Krankheiten werden immer seltener, dagegen nehmen chronische Leiden massiv zu. In den Industrieländern sind bereits 50\% der Erkrankten gleichzeitig von mehreren chronischen Krankheiten - der sogenanntem Multimorbidität - betroffen. Ein Grund, neue Wege zu gehen und neue Therapiekonzepte zu prüfen. Unter dem Thema «Tibetische Arzneimittel in der modernen Medizin» organisierte das UniversitätsSpital Zürich Ende September eine Konferenz für Ärzte und Apotheker in Zürich.

Einzelstrukturen wie etwa bestimmte Rezeptoren, sondern ganze funktionelle Felder verstärken oder schwächen könnten.

Zum Verständnis solcher Pflanzenrezepturen erforscht der Biochemiker Ao. Univ.-Prof. Florian Überall vom Biozentrum der Medizinischen Universität Innsbruck komplexe tibetische Arzneimittel auch im Hinblick auf die zu erwartenden Mehrfach-Wirkungen. So werden z.B. ORAC (Oxygen Radical Absorbance Capacity), ROS (Reactive Oxygen Species) und Zell-Detoxifikation untersucht. Dabei kommen auch Methoden wie Transkriptom- und Proteomanalyse sowie datenbankgestützte Netzwerkanalysen zum Einsatz, mit denen eine Beeinflussung der Abschrift der Geninformation gemessen wird. Prof. Überall zeigte in seiner neuesten Arbeit, dass bei dem tibetischen Arzneimittel Padma 28 rund 580 Transkriptionsereignisse moduliert wurden. Die entsprechenden Gene gehören zu 18 Netzwerkgruppen, in deren Zentrum die Funktionsgruppe «Herz-Kreislauf-System/Immunantwort» steht. «Das Ergebnis bestätigt nicht nur die Indikation des Arzneimittels (Durchblutungsstörungen), sondern gibt auch Aufschluss über den erweiterten Wirkkreis der Formel», erklärte Überall.
Auch klassische experimentelle Forschungsergebnisse wurden vorgestellt. Dr. Cécile Vennos, Leiterin Medizin und Wissenschaft bei der Padma AG, präsentierte eine Studie aus dem Inselspital Bern. Dabei wurde der Effekt von zwei anderen tibetischen Arzneimitteln auf die gastrointestinale Motilität untersucht. Die Ergebnisse zeigen differenzierte und modulierende Effekte von Padma Digestin auf die Magen- und Dünn-

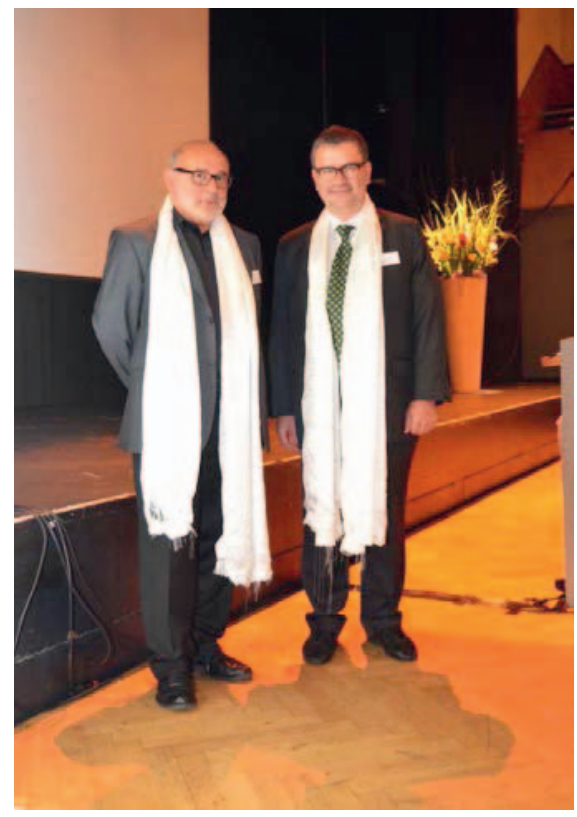

Abb. 1. Prof. Dr. med. Reinhard Saller und Dr. techn Herbert Schwabl wurden mit einem Khata (tibetischer Seidenschal) geehrt.
๑) 2012 S. Karger GmbH, Freiburg 


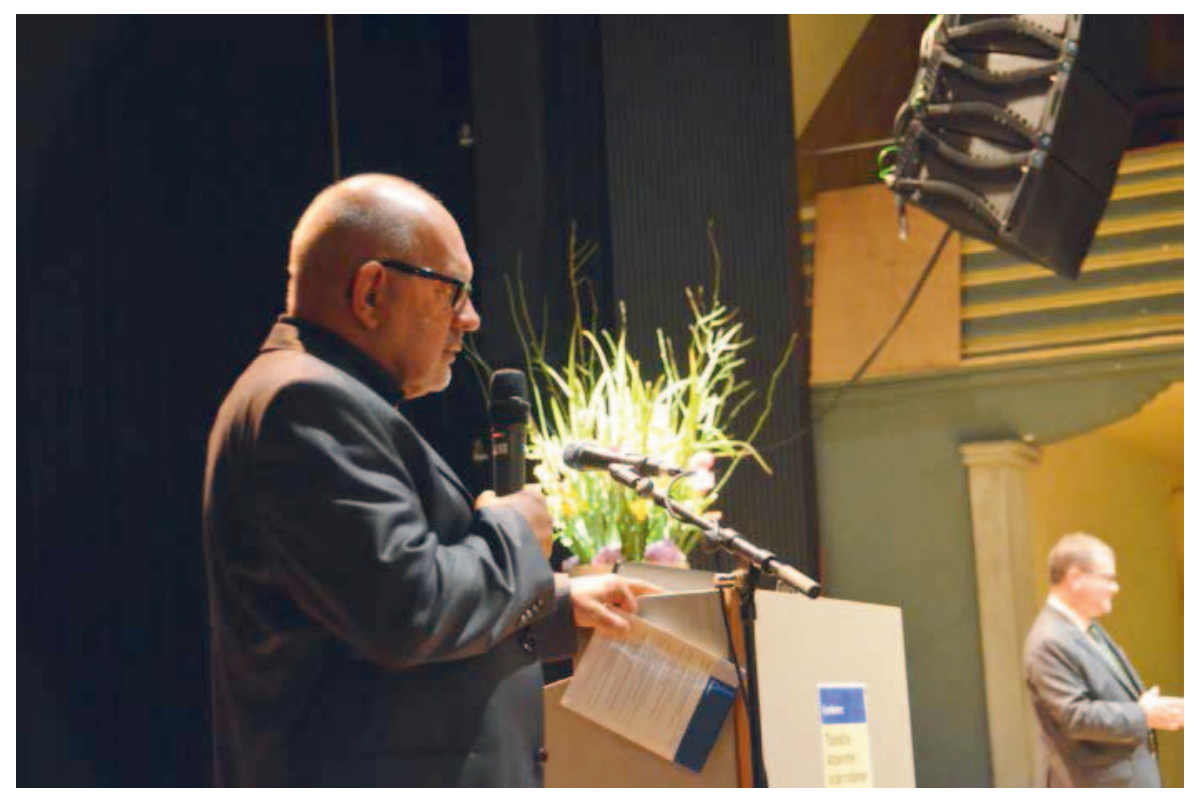

Abb. 2. Prof. Dr. med. Reinhard Saller und Dr. techn. Herbert Schwabl bei der Diskussionsrunde.

darmmotilität und von Padma Lax auf die Dickdarmmotorik. Die Resultate stützen damit die traditionellen Einsatzgebiete von Padma Digestin bei funktionellen Verdauungsstörungen und von Padma Lax bei Obstipation und obstipationsdominantem Reizdarmsyndrom.

Integration in die angiologische Praxis bei peripherer arterieller Verschlusskrankheit

Neben einer Beschreibung der Wirkmechanismen stand an der Tagung im Volkshaus Zürich vor allem die Praxis im Vordergrund. Dr. med. Christian Regli, leitender Arzt für konservative Angiologie am Kantonsspital Aarau, beschrieb die Wirkung der tibetischen Formel Padmed Circosan (Padma 28) auf den atherosklerotischen Formenkreis. «Wir setzen Padma Circosan zusätzlich zur herkömmlichen Standardtherapie ein. Dies vor allem bei Patienten, welche ein Gehtraining wegen der sogenannten «Schaufensterkrankheit» absolvieren. Wir haben damit gute Erfahrungen gemacht, welche auch in Studien nachgewiesen werden konnten», erklärte Regli. Jedoch sei der
Weitere Anwendungsstudien und Fallberichte

Die neueste klinische Studie mit einem tibetischen Arzneimittel stellte Prof. Dr. Rémy Meier, leitender Arzt der Gastroenterologie am Kantonsspital Liestal, vor. Untersucht wurden die Wirksamkeit, Verträglichkeit und Sicherheit der tibetischen Formel Se 'bru 5, in der Schweiz als Padma Digestin zugelassen, bei funktioneller Dyspepsie. Im Laufe der 6-wöchigen Einnahmedauer kam es zu einer hochsignifikanten Verbesserung des individuell am gravierendsten empfundenen Symptoms sowie von Beschwerden wie Völlegefühl nach dem Essen und Übelkeit $(p<0,01)$. Eine signifikante Besserung trat bei den Symptomen Magenkrämpfe, Schmerz in der Magengrube sowie Appetitmangel auf $(p<0,05)$. Bei Hungerund Nachtschmerz sowie Verstopfung konnte ebenfalls eine positive Veränderung nachgewiesen werden. Insgesamt beurteilten $81 \%$ der Ärzte und $76 \%$ der Patienten die Verträglichkeit als gut bis sehr gut. Von $21 \mathrm{~Pa}-$ tienten, die den Vergleich mit einer früheren Behandlung ziehen konnten, beurteilten 45\% die Therapie mit Padma Digestin als besser verträglich und $62 \%$ als wirksamer als die Vorbehandlung. Es kam zu keinen Veränderungen von Laborwerten oder Vitalzeichen. Bei 11 Patienten traten 17 unerwünschte Ereignisse auf, wobei bei Blähungen (zweimal), Juckreiz und Verstopfung (je einmal) ein $\mathrm{Zu}$ sammenhang mit der Studienmedikation als wahrscheinlich beurteilt wurde. Prof. Meier stellte der tibetischen Rezeptur ein gutes Zeugnis aus und bezeichnete sie als sinnvolle Erweiterung des bisherigen Therapieschemas.

Dr. med. Bettina Kneip, Institut für Naturheilkunde der Universität Zürich, stellte zwei Fälle aus der Praxis vor. Eine 54-jährige Patientin, die nach Mammakarzinom und antihormoneller Behandlung seit etwa 1,5 Jahren an Beschwerden wie nächtlichen Hitzewallungen, Durchschlaf- 
störungen, Stimmungsschwankungen usw. litt, klagte zusätzlich über kardiale Störungen wie rezidivierende Palpationen sowie schnelle Pulsbeschleunigung bei kleinsten Anstrengungen. Die Vorbehandlung bestand aus einem Betablocker sowie Weissdorn und Terminalia arjuna. Die Phytotherapeutika wurden nach 6 Wochen wegen mangelnder Wirkung abgesetzt und es wurde Padma Nerventonikum $(2 \times 1$ Kapsel $)$ verschrieben. Nach ca. 4 Wochen hatten sich die menopausalen wie auch die kardialen Beschwerden deutlich gebessert. Der Betablocker konnte ausgeschlichen werden. Der zweite Fall betraf den Einsatz von Padma Circosan bei Zytostatika-induzierten Dysästhesien und Ödemen der Hände bei einer 46-jährigen Patientin. Bereits nach 2 Wochen gingen die Dysästhesien deutlich zurück; nach 5 Wochen war die Patientin frei von Empfindungsstörungen und Ödemen.

Fazit

Die Fallberichte sowie die klinischen Studien zeigten, dass tibetische Arzneimittel inzwischen in zahlreichen schulmedizinischen Fachrichtungen angewendet werden. Neben der Angiologie sind dies auch die Gastroenterologie, Gynäkologie, Onkologie, Psychiatrie sowie die Zahnheilkunde. Durch die multimodale Wirkungsweise sowie die ausgeklügelte $\mathrm{Zu}$ sammensetzung bieten sich tibetische
Vielstoffgemische vor allem bei Therapien von Krankheiten mit komplexen Ursachen sowie bei chronischen Krankheiten an. So ist Prof. Dr. med. Reinhard Saller überzeugt, dass tibetische Arzneimittel, die sich aus Pflanzen und Mineralien zusammensetzen, innovative Therapieansätze darstellen. «Wir werden uns in Zukunft vermehrt mit multimorbiden Patienten auseinandersetzen müssen», sagt der Direktor des Instituts für Naturheilkunde des UniversitätsSpitals Zürich. Multifaktorielle Arzneimittel, wie sie die Tibetische Medizin kennt, stellen hier einen wertvollen Therapieansatz dar und sind auch in konventionelle «schulmedizinische» Behandlungskonzepte integrierbar. 